\title{
Search for a strongly decaying neutral charmed pentaquark
}

\author{
The FOCUS Collaboration ${ }^{1}$
}

J. M. Link ${ }^{a}$ P. M. Yager ${ }^{a}$ J. C. Anjos ${ }^{b}$ I. Bediaga ${ }^{b}$ C. Castromonte ${ }^{b}$ A. A. Machado ${ }^{b}$ J. Magnin ${ }^{b}$ A. Massafferri ${ }^{b}$ J. M. de Miranda ${ }^{b}$ I. M. Pepe ${ }^{b}$ E. Polycarpo ${ }^{b}$ A. C. dos Reis ${ }^{b}$ S. Carrillo ${ }^{c}$ E. Casimiro ${ }^{c}$ E. Cuautle ${ }^{c}$ A. Sánchez-Hernández ${ }^{c}$ C. Uribe ${ }^{c}$ F. Vázquez ${ }^{c}$ L. Agostino ${ }^{d}$ L. Cinquini ${ }^{d}$ J. P. Cumalat ${ }^{d}$ B. O'Reilly ${ }^{d}$ I. Segoni ${ }^{d}$ K. Stenson ${ }^{d}$ J. N. Butler ${ }^{\mathrm{e}} \mathrm{H}$. W. K. Cheung ${ }^{\mathrm{e}}$ G. Chiodini ${ }^{\mathrm{e}}$ I. Gaines ${ }^{\mathrm{e}}$ P. H. Garbincius ${ }^{\mathrm{e}}$ L. A. Garren ${ }^{\mathrm{e}}$ E. Gottschalk ${ }^{\mathrm{e}}$ P. H. Kasper ${ }^{\mathrm{e}}$ A. E. Kreymer ${ }^{\mathrm{e}}$ R. Kutschke ${ }^{\mathrm{e}}$ M. Wang ${ }^{\mathrm{e}}$ L. Benussi ${ }^{\mathrm{f}}$ M. Bertani ${ }^{f}$ S. Bianco ${ }^{f}$ F. L. Fabbri ${ }^{f}$ S. Pacetti ${ }^{f}$ A. Zallo ${ }^{f}$ M. Reyes ${ }^{g}$ C. Cawlfield ${ }^{\text {h }}$ D. Y. Kim ${ }^{h}$ A. Rahimi ${ }^{h}$ J. Wiss ${ }^{h}$ R. Gardner ${ }^{i}$ A. Kryemadhi ${ }^{i}$ Y. S. Chung ${ }^{j}$ J. S. Kang ${ }^{j}$ B. R. Ko ${ }^{\mathrm{j}}$ J. W. Kwak ${ }^{\mathrm{j}}$ K. B. Lee ${ }^{\mathrm{j}}$ K. Cho ${ }^{\mathrm{k}}$ H. Park ${ }^{\mathrm{k}}$ G. Alimonti ${ }^{\ell}$ S. Barberis ${ }^{\ell}$ M. Boschini ${ }^{\ell}$ A. Cerutti ${ }^{\ell}$ P. D'Angelo ${ }^{\ell}$ M. DiCorato ${ }^{\ell}$ P. Dini ${ }^{\ell}$ L. Edera ${ }^{\ell}$ S. Erba ${ }^{\ell}$ P. Inzani ${ }^{\ell}$ F. Leveraro ${ }^{\ell} \mathrm{S}$. Malvezzi ${ }^{\ell}$ D. Menasce ${ }^{\ell}$ M. Mezzadri ${ }^{\ell}$ L. Moroni ${ }^{\ell}$ D. Pedrini ${ }^{\ell}$ C. Pontoglio ${ }^{\ell}$ F. Prelz ${ }^{\ell}$ M. Rovere ${ }^{\ell}$ S. Sala ${ }^{\ell}$ T. F. Davenport III ${ }^{\mathrm{m}}$ V. Arena ${ }^{\mathrm{n}}$ G. Boca ${ }^{\mathrm{n}}$ G. Bonomi ${ }^{n}$ G. Gianini ${ }^{n}$ G. Liguori ${ }^{n}$ D. Lopes Pegna ${ }^{n}$ M. M. Merlo ${ }^{n}$ D. Pantea ${ }^{n}$ S. P. Ratti ${ }^{n}$ C. Riccardi ${ }^{n}$ P. Vitulo ${ }^{n}$ C. Göbel ${ }^{o}$ H. Hernandez ${ }^{p}$ A. M. Lopez ${ }^{p}$ H. Mendez ${ }^{p}$ A. Paris ${ }^{p}$

J. Quinones ${ }^{\mathrm{p}}$ J. E. Ramirez ${ }^{\mathrm{p}}$ Y. Zhang ${ }^{\mathrm{p}}$ J. R. Wilson ${ }^{\mathrm{q}}$ T. Handler ${ }^{r}$ R. Mitchell ${ }^{r}$ D. Engh ${ }^{\mathrm{s}}$ M. Hosack ${ }^{\mathrm{s}}$ W. E. Johns ${ }^{\mathrm{s}}$ E. Luiggi ${ }^{\mathrm{s}}$ J. E. Moores ${ }^{\mathrm{s}}$ M. Nehring ${ }^{\mathrm{s}}$ P. D. Sheldon ${ }^{\mathrm{s}}$ E. W. Vaandering ${ }^{\mathrm{s}}$ M. Webster ${ }^{\mathrm{s}}$ M. Sheaff ${ }^{\mathrm{t}}$

${ }^{a}$ University of California, Davis, CA 95616

${ }^{\mathrm{b}}$ Centro Brasileiro de Pesquisas Físicas, Rio de Janeiro, RJ, Brazil ${ }^{\mathrm{C}}$ CINVESTAV, o7000 México City, DF, Mexico 


\author{
d University of Colorado, Boulder, CO 80309

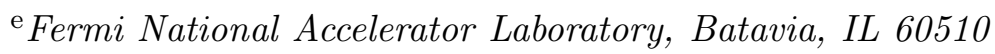 \\ ${ }^{\mathrm{f}}$ Laboratori Nazionali di Frascati dell'INFN, Frascati, Italy I-00044 \\ ${ }^{g}$ University of Guanajuato, 37150 Leon, Guanajuato, Mexico \\ ${ }^{\mathrm{h}}$ University of Illinois, Urbana-Champaign, IL 61801 \\ ${ }^{\mathrm{i}}$ Indiana University, Bloomington, IN 47405 \\ ${ }^{\mathrm{j}}$ Korea University, Seoul, Korea 136-701 \\ ${ }^{\mathrm{k}}$ Kyungpook National University, Taegu, Korea 702-701 \\ ${ }^{\ell}$ INFN and University of Milano, Milano, Italy \\ ${ }^{\mathrm{m}}$ University of North Carolina, Asheville, NC 28804 \\ ${ }^{\mathrm{n}}$ Dipartimento di Fisica Nucleare e Teorica and INFN, Pavia, Italy \\ ${ }^{\circ}$ Pontifícia Universidade Católica, Rio de Janeiro, RJ, Brazil \\ p University of Puerto Rico, Mayaguez, PR 00681 \\ ${ }^{\mathrm{q}}$ University of South Carolina, Columbia, SC 29208 \\ ${ }^{\mathrm{r}}$ University of Tennessee, Knoxville, TN 37996 \\ ${ }^{\mathrm{s}}$ Vanderbilt University, Nashville, TN 37235 \\ ${ }^{\mathrm{t}}$ University of Wisconsin, Madison, WI 53706
}

\begin{abstract}
We present a search for a charmed pentaquark decaying strongly to $D^{(*)-} p$. Finding no evidence for such a state, we set limits on the cross section times branching ratio relative to $D^{*-}$ and $D^{-}$under particular assumptions about the production mechanism.
\end{abstract}

Key words:

PACS: 14.80.-j 13.60.Le

\title{
1 Introduction
}

At the dawn of the QCD era, Jaffe proposed the existence of bound (mass below threshold for strong decay) multiquark states including $Q \bar{Q} q \bar{q}$ states and the $H$ dihyperon [1] based on calculations using the bag model [2]. Calculations a decade later $[3,4,5]$ indicated that a $S=-1$ charmed pentaquark $(\bar{c} s q q q)$ is more likely to be bound than either the $H$ dihyperon or a $S=0$ charmed pentaquark ( $\bar{c} q q q q)$ due to the lack of a quark exchange process in

${ }_{1}^{1}$ See http://www-focus.fnal.gov/authors.html for additional author information. 
the lowest decay channel, $D_{s}^{-} N^{2}$. Additional calculations using bag models and group theory assuming a one-gluon exchange interaction suggested that $S=0,-1$ charmed pentaquarks were probably unbound due to $\mathrm{SU}(3)$ symmetry breaking and the finite mass of the charm quark $[6,7,8]$. Calculations using an instanton model indicated a nearly bound $S=-1$ charmed pentaquark [9] while calculations based on Goldstone boson exchange predicted unbounded $S=-1$ charm pentaquarks but allowed bounded or unbounded $S=0$ charm pentaquarks [10,11]. Finally, the Skyrme (chiral soliton) models predicted deeply bound charmed pentaquark states with $S=0,-1[12,13,14]$. Searches in 1998 and 1999 found no evidence for a weakly decaying charmed $S=-1$ pentaquark from $\pi^{-} N$ interactions [15].

The pentaquark field underwent a transformation between January 2003 and March 2004, when no less than ten independent pentaquark observations at a mass around $1540 \mathrm{MeV} / \mathrm{c}^{2}$ were reported [16], with a presumed quark content of $(\bar{s} u u d d)$. This triggered a rash of theoretical explanations, including predictions of charmed pentaquark states based on various quark models [17], Skyrme models [18], and lattice QCD [19] which predicted $\Theta_{c}^{0}(\bar{c} u u d d)$ masses of $2600-3000 \mathrm{MeV} / c^{2}, 2700 \mathrm{MeV} / c^{2}$, and $3450 \mathrm{MeV} / c^{2}$, respectively, compared to the $D N$ threshold of $\sim 2805 \mathrm{MeV} / c^{2}$. In March 2004, the H1 Collaboration reported evidence for a $S=0$ charmed pentaquark state decaying to $D^{*-} p$ at a mass of $3099 \pm 3 \pm 5 \mathrm{MeV} / c^{2}$ and a statistical significance of $5.4 \sigma$ or $6.2 \sigma$, depending on how the significance is calculated [20]. This precipitated additional theoretical effort into pentaquark states with a heavy quark [21].

This letter presents a search for the $\Theta_{c}^{0}(\bar{c} u u d d)$ pentaquark candidate. We search using the same decay mode as $\mathrm{H} 1\left(\Theta_{c}^{0} \rightarrow D^{*-} p\right)$ and the other obvious decay mode, $\Theta_{c}^{0} \rightarrow D^{-} p$. Since the $D^{*+}$ statistics and data quality of the FOCUS experiment are much better than the observing experiment, and the production mechanism is similar, we should be able to confirm or refute the existence of the purported state.

\section{Event reconstruction and selection}

The FOCUS experiment recorded data during the 1996-7 fixed-target run at Fermilab. A photon beam obtained from bremsstrahlung of $300 \mathrm{GeV}$ electrons and positrons impinged on a set of BeO targets. Four sets of silicon strip detectors, each with three views, were located downstream of the targets for vertexing and track finding. For most of the run, two pairs of silicon strips were also interleaved with the target segments for more precise vertexing [22]. Charged particles were tracked and momentum analyzed as they

$\overline{2}$ Charged conjugate states are implied unless explicitly stated otherwise 
passed through one or two dipole magnets and three to five sets of multiwire proportional chambers with four views each. Three multicell threshold Čerenkov counters, two electromagnetic calorimeters, and two muon detectors provided particle identification. A hadronic trigger passed 6 billion events for reconstruction. The average photon energy of reconstructed charm events is $175 \mathrm{GeV}$.

A candidate driven vertexing algorithm is used to reconstruct charm. In the case of $D^{0} \rightarrow K^{-} \pi^{+}, K^{-} \pi^{+} \pi^{-} \pi^{+}\left(D^{+} \rightarrow K^{-} \pi^{+} \pi^{+}\right)$, the charged tracks are required to verticize with $\mathrm{CL}>2 \%(1 \%)$ with the correct summed charge. The momentum and vertex location are used as a "seed" track to find the production vertex, which must have CL $>1 \%$. The $D^{*+}$ candidate is obtained from the decay $D^{*+} \rightarrow D^{0} \pi_{s}^{+}$. The soft pion must be consistent with originating from the production vertex and the track is refit using the production vertex as an extra constraint.

The selection criteria were chosen to maximize $S / \sqrt{B}$. The signal, $S$, comes from a Monte Carlo simulation. Since the production characteristics of the pentaquark are unknown, we use PYTHIA to generate charm events with a $\Xi_{c}^{0}$. The $\Xi_{c}^{0}$ is produced at a mass of $2.47 \mathrm{GeV} / c^{2}$ but is promoted to a mass of $3.1 \mathrm{GeV} / c^{2}$ before decaying to the decay mode of interest (with zero lifetime). The background, $B$, is obtained from the wrong sign data $\left(D^{(*)+} p\right)$ over the entire mass range of study (threshold to $4 \mathrm{GeV} / c^{2}$ ). This is an unbiased method for determining the selection criteria.

Separating charm from hadronic background is primarily accomplished by requiring the decay vertex be distinct from the production vertex. A cut of $\ell / \sigma_{\ell}>2,4,6$ is applied to $D^{0} \rightarrow K^{-} \pi^{+}, D^{0} \rightarrow K^{-} \pi^{+} \pi^{-} \pi^{+}, D^{+} \rightarrow K^{-} \pi^{+} \pi^{+}$ where $\ell$ is the distance between the two vertices and $\sigma_{\ell}$ is the calculated uncertainty $\left(\left\langle\sigma_{\ell}\right\rangle \sim 500 \mu \mathrm{m}\right)$. Since hadronic reinteractions can fake a decay, requiring the decay vertex be located outside of target material reduces background. The out-of-material significance $\sigma_{\text {out }}$ is positive (negative) for a vertex outside (inside) material. We require $2 \ell / \sigma_{\ell}+\max \left(-2, \sigma_{\text {out }}\right)>4,8,12$ for $D^{0} \rightarrow K^{-} \pi^{+}, D^{0} \rightarrow K^{-} \pi^{+} \pi^{-} \pi^{+}, D^{+} \rightarrow K^{-} \pi^{+} \pi^{+}$. To ensure the $D^{0}$ decay tracks do not originate from the production vertex, a cut is made on the change in production vertex confidence level when any $D^{0}$ decay track is added to the vertex $\left(\Delta \mathrm{CL}_{\mathrm{pri}}\right)$. We require $\Delta \mathrm{CL}_{\mathrm{pri}}<30 \%(0)$ for $D^{0}\left(D^{+}\right)$ decays. Also, the transverse momentum of the $D$ candidate with respect to the line-of-flight obtained from the vertices must be less than $0.7,0.4$, and $0.3 \mathrm{GeV} / c$ for $D^{0} \rightarrow K^{-} \pi^{+}, D^{0} \rightarrow K^{-} \pi^{+} \pi^{-} \pi^{+}$, and $D^{+} \rightarrow K^{-} \pi^{+} \pi^{+}$, respectively. Since the signal contains a charged kaon, information from the three Čerenkov counters effectively suppresses backgrounds. The Čerenkov identification algorithm [23] returns negative log-likelihood (times two) values $\mathcal{W}_{i}(j)$ for track $j$ and hypothesis $i \in\{e, \pi, K, p\}$. In practice, differences in loglikelihoods between hypotheses are used such as $\Delta \mathcal{W}_{\pi K} \equiv \mathcal{W}_{\pi}-\mathcal{W}_{K}$. For the 

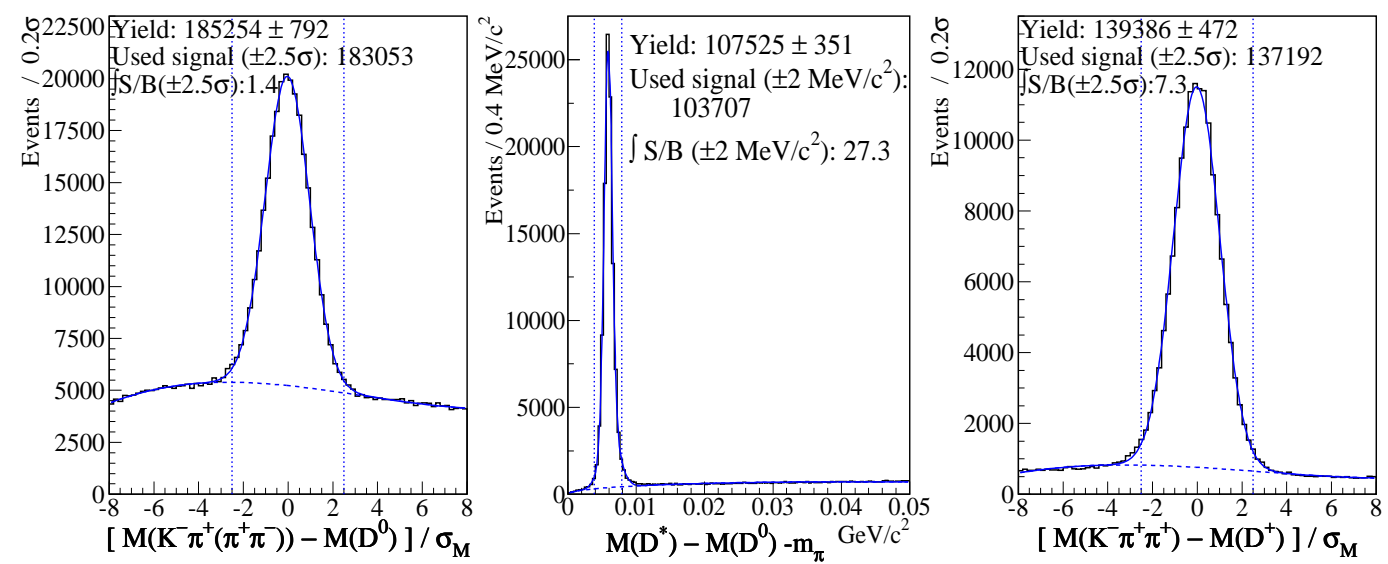

Fig. 1. The normalized mass plots of $D^{0} \rightarrow K^{-} \pi^{+}$and $D^{0} \rightarrow K^{-} \pi^{+} \pi^{-} \pi^{+}$candidates (left) and $D^{+} \rightarrow K^{-} \pi^{+} \pi^{+}$candidates (right) are fit with a single Gaussian for the signal and a quadratic polynomial for the background. The energy release plot for $D^{*+}$ candidates of $D^{0}$ events (middle) is fit with a double Gaussian for the signal plus a threshold function $\alpha Q^{1 / 2}+\beta Q^{3 / 2}$ for the background. Events inside the vertical lines are selected for analysis.

$D^{*-}$ candidates, we require $\Delta \mathcal{W}_{\pi K}(K)>0.5, \mathcal{W}_{\min }(\pi)-\mathcal{W}_{\pi}(\pi)>-5$, and $\mathcal{W}_{\min }\left(\pi_{s}\right)-\mathcal{W}_{\pi}\left(\pi_{s}\right)>-5$ where $\mathcal{W}_{\min } \equiv \min \left(\mathcal{W}_{i \in\{e, \pi, K, p\}}\right)$. For the $D^{+}$candidates, we require $\Delta \mathcal{W}_{\pi K}(K)>2$ and $\mathcal{W}_{\min }(\pi)-\mathcal{W}_{\pi}(\pi)>-2$. The remaining selection criteria are very efficient and mildly suppress some backgrounds. For the $D^{0} \rightarrow K^{-} \pi^{+}$decay, the momentum asymmetry, $\left|\frac{p(K)-p(\pi)}{p(K)+p(\pi)}\right|$, must be less than $\frac{p(D)+130 \mathrm{GeV} / c}{200 \mathrm{GeV} / c}$. The summed $p_{T}^{2}$ of $D$ daughters with respect to the $D$ momentum vector must be greater than $0.15 \mathrm{GeV}^{2} / c^{2}$. These last two cuts favor a decay of a heavy particle over combinatoric background. The charm signals obtained with these cuts are shown in Fig. 1 . We select $D^{0}$ and $D^{+}$candidates with an invariant mass within $2.5 \sigma_{M}$ of the nominal mass where $\sigma_{M}$ is the calculated error on the mass. We select $D^{*+}$ candidates within $2 \mathrm{MeV} / c^{2}$ of the nominal energy release, $Q \equiv M\left(D^{*+}\right)-M\left(D^{0}\right)-m_{\pi^{+}}$. These values were also chosen in the same optimization procedure. The proton candidate must be consistent with originating from the production vertex and must be strongly favored to be a proton by the Cerenkov systems; $\Delta \mathcal{W}_{\pi p}(p)>6$ and $\Delta \mathcal{W}_{K p}(p)>2$. Again, these criteria were selected in the unbiased manner described above.

\section{Pentaquark search results}

The $D^{*-} p$ and $D^{-} p$ invariant mass plots, separated by charge states and summed together, are shown in Fig. 2 with fitted background curves superimposed. The individual charge states show approximately an equal number of events in each state and so for the remainder of the analysis we combine the 

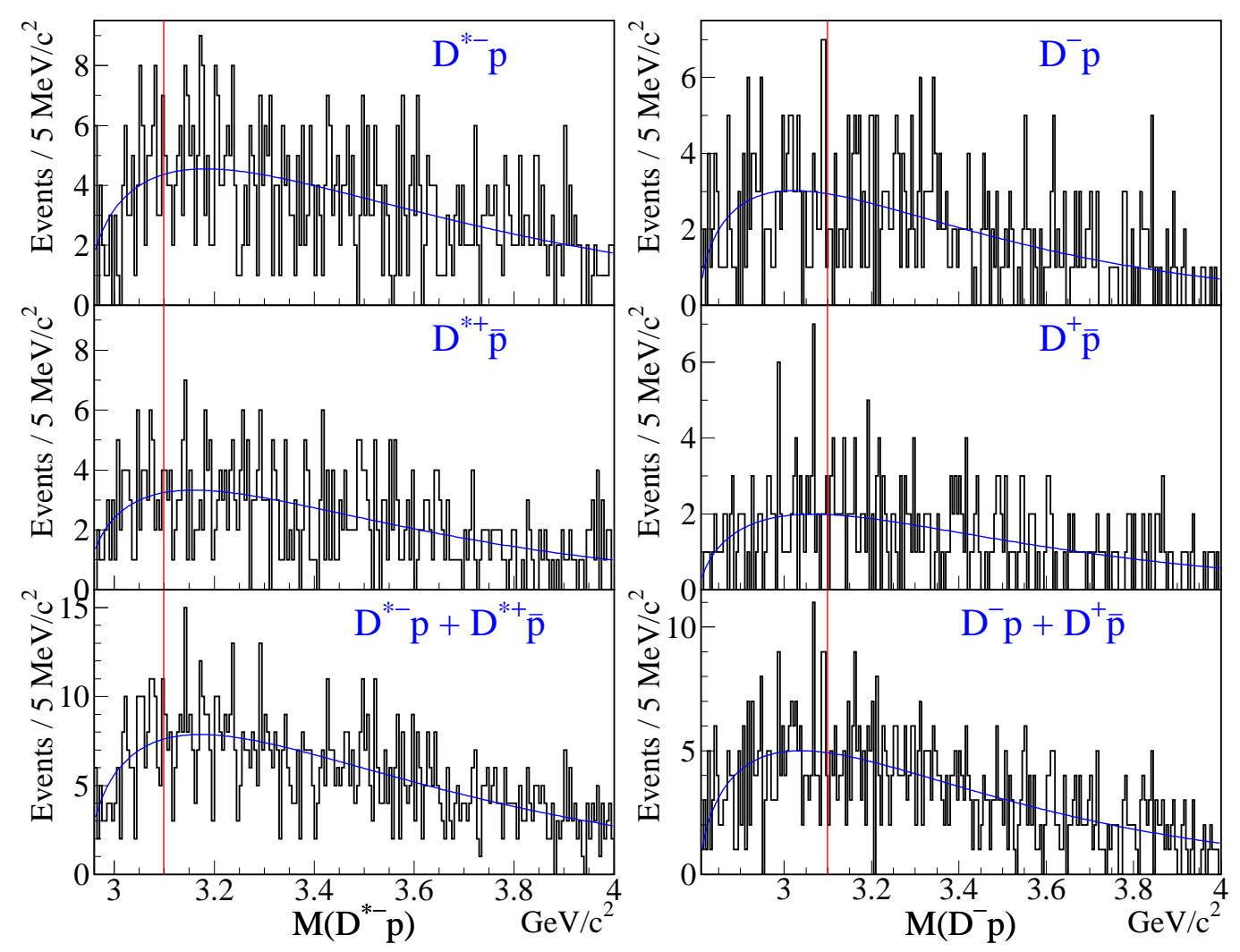

Fig. 2. Invariant mass plots of $D^{*-} p$ candidates (left) and $D^{-} p$ candidates (right). The top and middle plots show the $\bar{D} p$ and $D \bar{p}$ contributions, respectively. The bottom plot includes both charge conjugate states. Plots are fit to a background shape: $a Q^{b} \exp (c Q)$ where $Q \equiv M\left(\Theta_{c}\right)-M\left(D^{(*)-}\right)-m_{p}$ is the energy release.

charge conjugate states. No evidence for a pentaquark at $3.1 \mathrm{GeV} / c^{2}$ or at any mass less than $4 \mathrm{GeV} / c^{2}$ is observed. To set a limit on the yield we first need information about the width of the state. H1 reports a measured Gaussian width of $\sigma_{T}=12 \pm 3 \mathrm{MeV} / c^{2}$ with an expected resolution of $\sigma_{R}=7 \pm 2 \mathrm{MeV} / c^{2}$. We define the "natural" width as $\sigma_{N}=\sqrt{\sigma_{T}^{2}-\sigma_{R}^{2}}$. Since $\sigma_{T}$ and $\sigma_{R}$ agree at better than $95 \% \mathrm{CL}$, the lower limit on $\sigma_{N}$ is 0 . Using the above measurements to construct a $\chi^{2}$, we set $\chi^{2}=3.84$ to find the $95 \%$ CL upper limit on $\sigma_{N}$. The maximum value of $\sigma_{N}$ for $\chi^{2}=3.84$ occurs at $\sigma_{N}=16.6 \mathrm{MeV} / c^{2}$ with $\sigma_{R}=6.1 \mathrm{MeV} / c^{2}$ and $\sigma_{T}=17.7 \mathrm{MeV} / c^{2}$. We construct limits on yields under the two extreme assumptions about the pentaquark "natural" width, $\sigma_{N}=0$ and $\sigma_{N}=16.6 \mathrm{MeV} / c^{2}$. To a very good approximation, the upper limit increases monotonically with $\sigma_{N}$ so this provides the extreme range. In both cases, $\sigma_{N}$ is added in quadrature to the experimental resolution to obtain the total width, $\sigma_{T}$. The experimental resolution increases linearly from $2 \mathrm{MeV} / c^{2}$ at threshold to $14(13) \mathrm{MeV} / c^{2}$ at $3.95 \mathrm{GeV} / c^{2}$ for $D^{*-} p\left(D^{-} p\right)$.

The $D^{-} p\left(D^{*-} p\right)$ mass plots are fit in $1 \mathrm{MeV} / c^{2}$ steps from 2.84 (2.98) to $3.9 \mathrm{GeV} / c^{2}$. At each step, the mass and width of the Gaussian signal are fixed while the signal yield and background parameters are fitted. A binned log- 


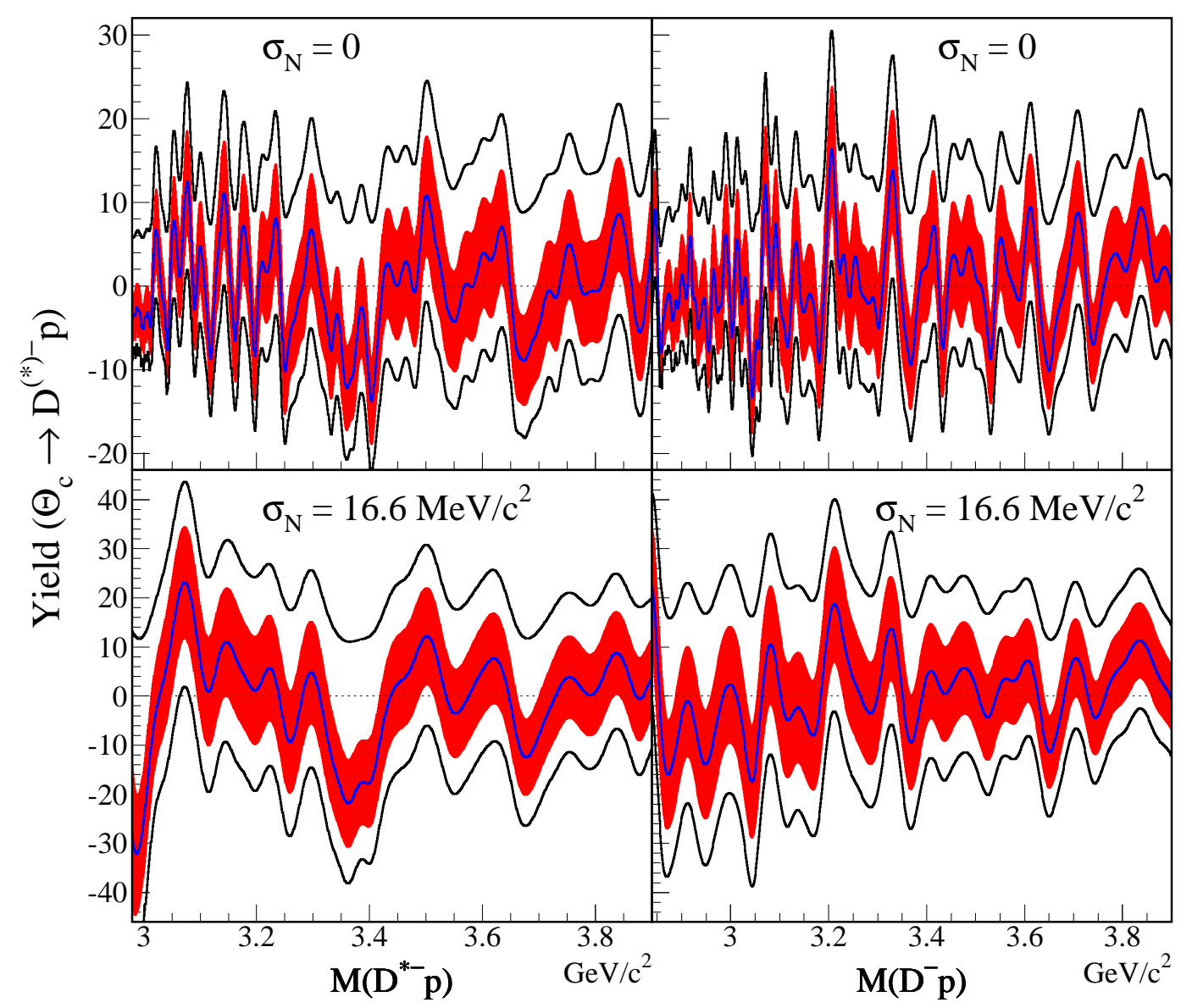

Fig. 3. Charmed pentaquark yields and upper limits. Left (right) plots show results for $\Theta_{c} \rightarrow D^{*-} p\left(\Theta_{c} \rightarrow D^{-} p\right)$. Top (bottom) plots show results for a natural width of $0\left(16.6 \mathrm{MeV} / c^{2}\right)$. The shaded region includes the $1 \sigma$ errors with the central value in the middle. The outer curves show the upper and lower limits.

likelihood fit using Minuit [24] is performed with a background shape given by $a Q^{b} \exp (c Q)$ where $Q$ is the energy release. The $1 \sigma$ error is defined as the point where $\Delta \log \mathcal{L}=0.50$ relative to the maximum $\log \mathcal{L}$, while continually adjusting the background parameters to maximize $\log \mathcal{L}$. The $95 \%$ CL lower limit is defined similarly with $\Delta \log \mathcal{L}=1.92$. Both are obtained using MINOS [24]. The 95\% CL upper limit is constructed as follows. The likelihood function $\mathcal{L}$ versus yield is determined by maximizing $\log \mathcal{L}$ for many different (fixed) yields, allowing background parameters to float. The likelihood function is integrated from a yield of 0 to $\infty$. The $95 \%$ CL upper limit on the yield is defined as the point where $95 \%$ of the total likelihood is between a yield of 0 and the upper limit. The fitted yield, $1-\sigma$ errors, and 95\% CL limits are shown in Fig. 3.

To compare with other experiments, the limits on yield must be converted to limits on production times (unknown) branching ratio. We choose to normalize the $\Theta_{c}^{0}$ production cross section to the $D$ meson production cross 
section from which it is reconstructed. That is, we attempt to determine $\sigma\left(\Theta_{c}^{0}\right) \cdot B R\left(\Theta_{c}^{0} \rightarrow D^{-} p\right) / \sigma\left(D^{-}\right)$and $\sigma\left(\Theta_{c}^{0}\right) \cdot B R\left(\Theta_{c}^{0} \rightarrow D^{*-} p\right) / \sigma\left(D^{*-}\right)$.

The FOCUS detector is a forward spectrometer and therefore acceptance depends on the produced particle momentum. The production characteristics of the charmed pentaquark is by far the largest source of systematic uncertainty in this analysis. We choose a particular production model to obtain limits and provide sufficient information about the experiment for other interested parties to obtain limits based on other production models. The production simulation begins with a library of $e^{-}$and $e^{+}$tracks obtained from a TURTLE simulation [25] of the Wideband beam line. From this library, an individual track is drawn and bremsstrahlung photons created by passage through a $20 \% X_{0}$ lead radiator. The charm cross section is then applied to the bremsstrahlung photons using Monte Carlo rejection. A photon which produces a charm event is sent to the Pythia event generator [26]. ${ }^{3}$ Since PyThia does not produce charmed pentaquarks, another particle must be chosen to represent the charmed pentaquark. Charmed baryons are the natural choices since they have a single charm quark like the charmed pentaquark and are closer to the correct number of total quarks than charmed mesons. Since it is possible to adjust the mass of the chosen charmed baryon in PYTHIA, it is not necessary to pick the charmed baryon with the highest mass. Other than mass, the most important effect on the production is the number of quarks a particle has in common with the initially interacting hadrons, due to the nature of the PYTHIA string fragmentation model. The $\Xi_{c}^{0}$ and $\Sigma_{c}^{+}$particles are chosen to represent the extremes in the production of a charmed pentaquark. Other than the charm quark, the $\Xi_{c}^{0}(c s d)\left(\Sigma_{c}^{+}(c u d)\right)$ can obtain at most $50 \%$ (100\%) of the remaining quarks from the target nucleon valence quarks, while the $\Theta_{c}^{0}(\bar{c} u u d d)$ can take $75 \%$. In all cases, the charge conjugate particles must obtain all quarks from the vacuum. The mass of the particle chosen to represent the pentaquark, $\Xi_{c}^{0}$ or $\Sigma_{c}^{+}$, is set to the appropriate value in PYTHIA, by setting PMAS $(190,1)$ or PMAS(187,1), respectively. This method differs from that used to generate the Monte Carlo sample for cut optimization since in this case the produced mass is changed to the appropriate value. For a photon which passes the charm cross section, the PYTHIA generator is run up to 1000 times searching for a $\Xi_{c}^{0}\left(\Sigma_{c}^{+}\right)$. This fails approximately $90 \%$ of the time, especially for low energy photons. When this happens, a new charm producing photon is selected. This changes the photon spectrum for pentaquark producing events. The ini-

3 PYTHIA version 6.127 is used with modifications to many parameters which provides a better match to the FOCUS charm data: $\operatorname{MSTP}(14): 20 \rightarrow 0, \operatorname{PARP}(2): 10 \rightarrow$ 3, PMAS(4,1): $1.35 \rightarrow 1.6, \operatorname{MSTJ}(21): 2 \rightarrow 0$, PARJ(1): $0.1 \rightarrow 0.3$, PARJ(3): $0.4 \rightarrow$ 0.25, MSTJ(12): $2 \rightarrow 4$, $\operatorname{MSTP}(92): 4 \rightarrow 2, \operatorname{PARP}(96): 3 \rightarrow 2, \operatorname{PARP}(97): 1 \rightarrow 2$, $\operatorname{PARP}(91): 0.44 \rightarrow 0.6, \operatorname{PARP}(93): 2 \rightarrow 3, \operatorname{PARP}(99): 0.44 \rightarrow 0.6, \operatorname{PARP}(100): 2 \rightarrow 3$, PARJ(21): $0.36 \rightarrow 0.425$, MSTJ(13): $0 \rightarrow 1$, PARJ(41): $0.3 \rightarrow 0.25$, PARJ(42): $0.58 \rightarrow$ $0.7, \operatorname{PARJ}(45): 0.5 \rightarrow 0.75, \operatorname{PARJ}(36): 2 \rightarrow 1$. 


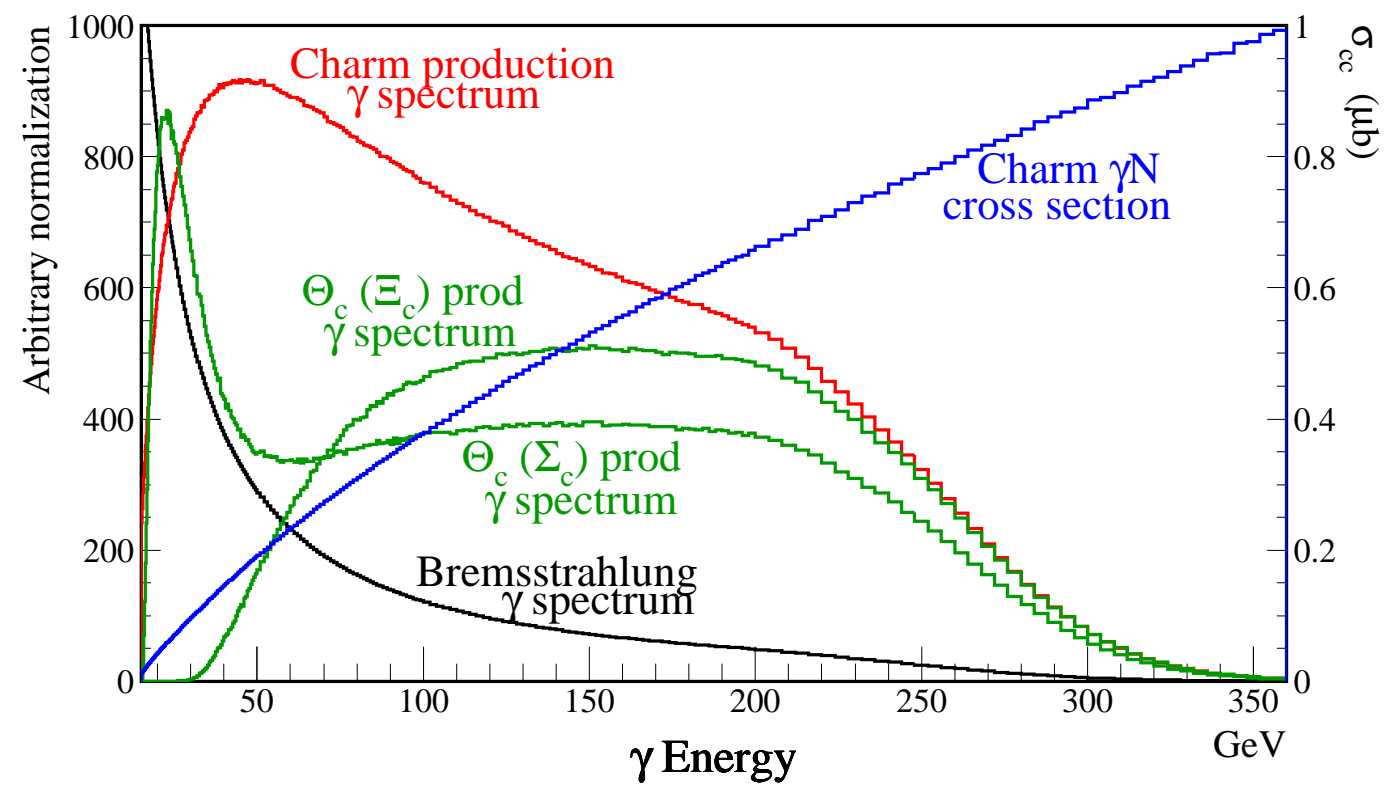

Fig. 4. The initial bremsstrahlung spectrum peaks at low energies. Applying the rising charm cross section results in a harder photon spectrum for charm inducing photons. The photons producing $3.1 \mathrm{GeV} / c^{2} \Xi_{c}$ and $\Sigma_{c}$ particles are harder than generic charm inducing photons except for a low energy peak for $\Sigma_{c}$ production due to the charm quark combining with quarks from the target nucleon.

tial bremsstrahlung spectrum, applied charm cross section, charm producing photon spectrum, and pentaquark producing photon spectra for $\Xi_{c}^{0}$ and $\Sigma_{c}^{+}$ are shown in Fig. 4. After production, the $\Xi_{c}^{0}$ or $\Sigma_{c}^{+}$is changed to the $\Theta_{c}^{0}$ with zero lifetime and forced to decay in the mode of interest. The momentum of the generated particles, the momentum of the reconstructed particles, and the ratio of the two (efficiency versus momentum) are shown in Fig. 5 for a pentaquark mass of $3.1 \mathrm{GeV} / c^{2}$. Since there are many low momentum pentaquarks from $\Sigma_{c}^{+}$which are not reconstructed, the overall efficiency for pentaquarks produced as $\Sigma_{c}^{+}$is lower than for pentaquarks produced as $\Xi_{c}^{0}$. The low generated momentum spike for pentaquarks produced as $\Sigma_{c}^{+}$is a result of the produced charm quark combining with a $u$ and a $d$ quark from the target. The efficiency versus mass is shown in Fig. 6 where the top (bottom) curve shows the efficiency for pentaquarks produced as $\Xi_{c}^{0}\left(\Sigma_{c}^{+}\right)$.

The limits on $\Theta_{c}^{0}$ production are made relative to $D^{(*)-}$ production. Therefore, the efficiency for $D^{*-}$ and $D^{-}$must be determined. PyтHIA is again used to model charm production. In this case we have verified that PYTHIA correctly models charm production in the region over which we have acceptance. This is less than $10 \%$ of the total charm produced. Note, however, that only the ratio of efficiencies between $\Theta_{c}^{0}$ and $D^{(*)-}$ production need be determined. Therefore, discrepancies between PyтнiA and reality in the low momentum (unobserved) region are likely to cancel. The global efficiency for $D^{*-}\left(D^{-}\right)$ reconstruction is $2.81 \%(3.05 \%)$. 


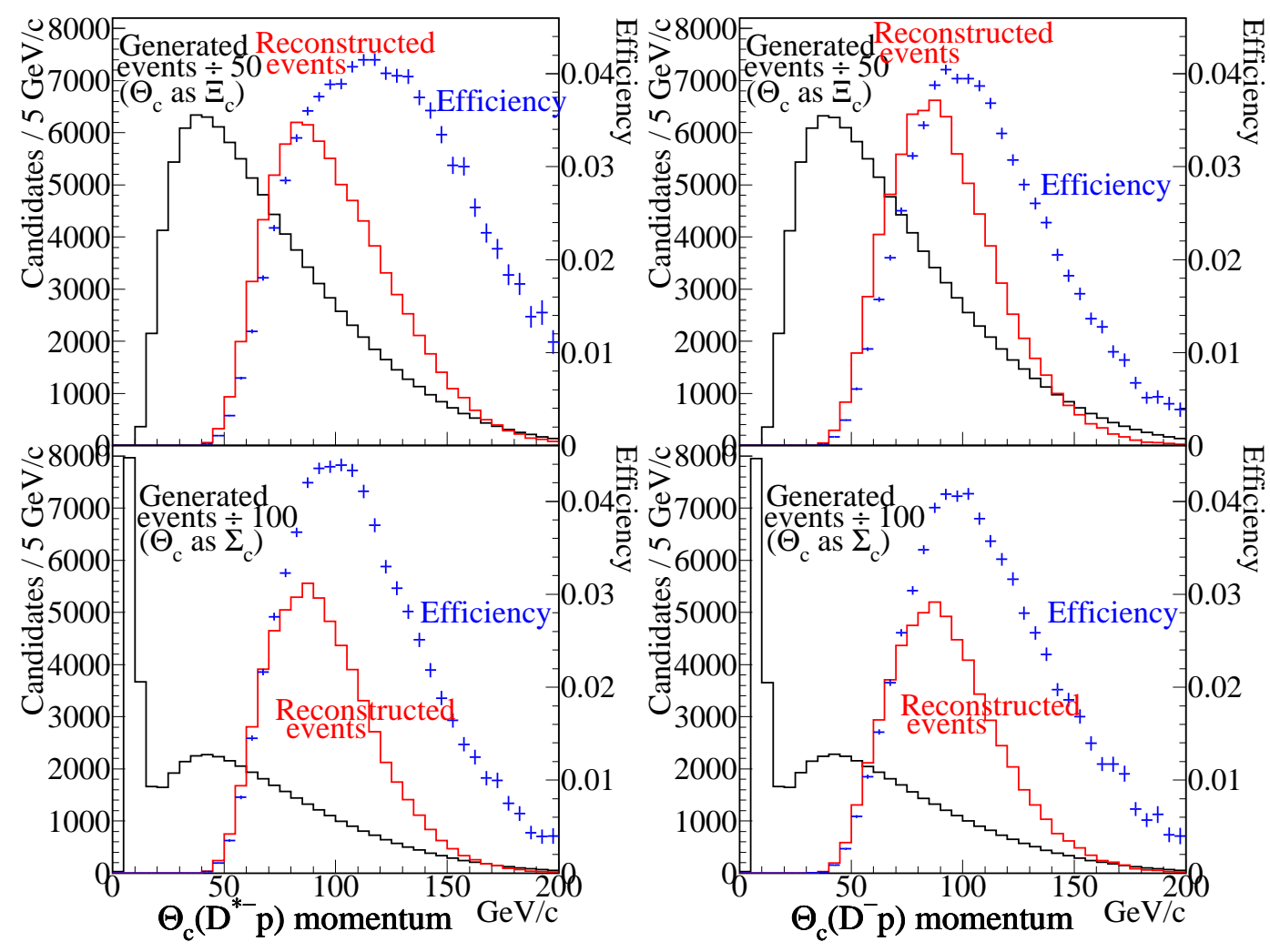

Fig. 5. All four plots show generated and reconstructed events (histograms) and the calculated efficiency (points with error bars) versus pentaquark momentum. The top (bottom) plots are for pentaquarks produced as $\Xi_{c}^{0}\left(\Sigma_{c}^{+}\right)$. The left (right) plots are for the decay $\Theta_{c}^{0} \rightarrow D^{*-} p\left(\Theta_{c}^{0} \rightarrow D^{-} p\right)$.
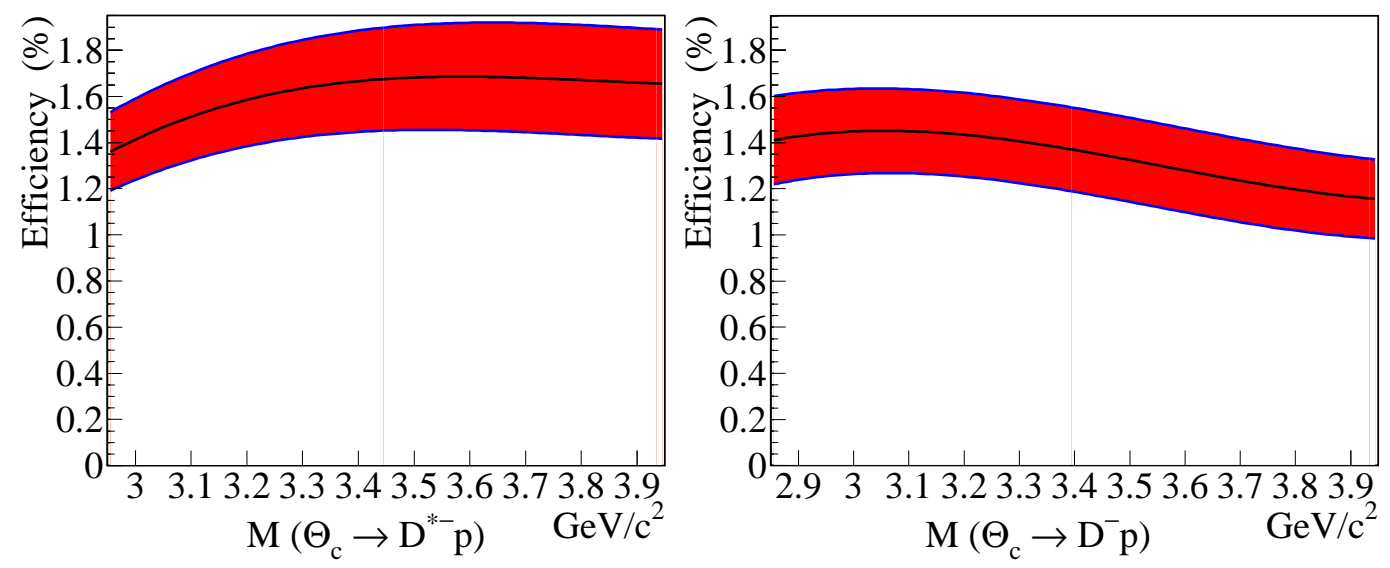

Fig. 6. Efficiency (including acceptance) versus mass for reconstructing $\Theta_{c}^{0} \rightarrow D^{*-} p$ (left) and $\Theta_{c}^{0} \rightarrow D^{-} p$ (right). The top (bottom) curves are obtained from pentaquarks produced as $\Xi_{c}^{0}\left(\Sigma_{c}^{+}\right)$while the middle curve is the average.

We attempt to determine:

$$
\frac{\sigma\left(\Theta_{c}^{0} \rightarrow D p\right) \cdot \mathrm{BR}\left(\Theta_{c}^{0} \rightarrow D p\right)}{\sigma(D)}=\frac{Y_{\Theta_{c}^{0}}}{\epsilon_{\Theta_{c}^{0}}} \frac{\epsilon_{D}}{Y_{D}}
$$


where $D$ is either $D^{*-}$ or $D^{-}$. The $D$ yield $\left(Y_{D}\right)$ and pentaquark yield $\left(Y_{\Theta_{c}^{0}}\right)$ are obtained from Fig. 1 and Fig. 3, respectively. The uncertainty on the $D$ yield is less than 1\%, insignificant compared to other uncertainties in the analysis. The remaining quantity is the ratio of efficiencies, $\epsilon_{D} / \epsilon_{\Theta_{c}^{0}}$. Our uncertainty in $\epsilon_{D}$ is much less than $\epsilon_{\Theta_{c}^{0}}$ since the Monte Carlo simulation uses a tuned version of Pythia which accurately reproduces the $D$ production for the region in which the FOCUS detector has acceptance. The dominant source of systematic uncertainty comes from a lack of knowledge of the pentaquark production characteristics which is reflected in $\epsilon_{\Theta_{c}^{0}}$. An estimate of this systematic uncertainty is obtained from Fig. 6 which shows the efficiency for $\Theta_{c}^{0}$ produced as $\Xi_{c}^{0}$ (top curve), $\Sigma_{c}^{+}$(bottom curve), and the average (middle curve). We take the average as our central value for the efficiency $\left(\epsilon_{0}\right)$ and the difference between the average and top (or bottom) curve as the $1 \sigma$ error on the efficiency $\left(\sigma_{\epsilon_{0}}\right)$. To include the error we fit directly for the corrected yield $Y_{\Theta_{c}^{0}} / \epsilon_{\Theta_{c}^{0}}$, include the efficiency as a free parameter and use a modified log-likelihood: $\log \mathcal{L}^{\prime}=\log \mathcal{L}-0.5\left(\epsilon_{\text {fit }}-\epsilon_{0}\right)^{2} / \sigma_{\epsilon_{0}}^{2}$ where $\epsilon_{\text {fit }}$ is the fitted efficiency which is always equal to $\epsilon_{0}$ when $\log \mathcal{L}^{\prime}$ is maximized. The corrected yield, $1 \sigma$ errors, and 95\% CL upper limit is obtained in the same manner as described previously for the uncorrected yield and is shown in Fig. 7.

\section{Conclusions}

FOCUS has published the most precise measurements of the mass and width of four $D^{* *}$ states [27] with decay modes similar to that of the charmed pentaquark. However, from a FOCUS data set of 104,000 $D^{*-}$ and $137,000 D^{-}$de-

cays, we find no evidence for a charm pentaquark decaying strongly to $D^{(*)-} p$ over the mass range of $30 \mathrm{MeV} / c^{2}$ above threshold to $3.9 \mathrm{GeV} / c^{2}$. Results for yields and cross sections versus mass are found in Figs. 3 and 7. A summary of these limits over the entire mass range is given in Table 1. The sample of $D^{*-}$ events is more than 30 times larger as well as cleaner than the sample used by $\mathrm{H} 1$ in the paper which found evidence for the charm pentaquark [20]. While $\mathrm{H} 1$ finds $\sim 1 \%$ of the $D^{*-}$ from $\Theta_{c}^{0}$, FOCUS sets a limit of $<0.075 \%$ at 95\% CL. In addition, the production is similar between the two experiments; virtual (real) photons on protons (nucleons) for H1 (FOCUS). Thus, the H1 result is either a statistical fluctuation or the result of an unusual production mechanism which increases the charm pentaquark to charm cross section by a factor of at least 10 in $\mathrm{H} 1$ relative to FOCUS. A simple study to determine the extent of the difference was performed. PYTHIA was used to generate $D^{*+}$ and $\Theta_{c}^{0}(3100)$ events using production appropriate for FOCUS and H1. The $\Theta_{c}^{0}(3100)$ was modeled as the average of $\Xi_{c}^{0}$ and $\Sigma_{c}^{+}$, both at $3.1 \mathrm{GeV} / c^{2}$. Only events in the acceptance of the experiment were accepted by requiring the pentaquark momentum be greater than $50 \mathrm{GeV} / c$ for FOCUS and requiring $\mathrm{H} 1$ 


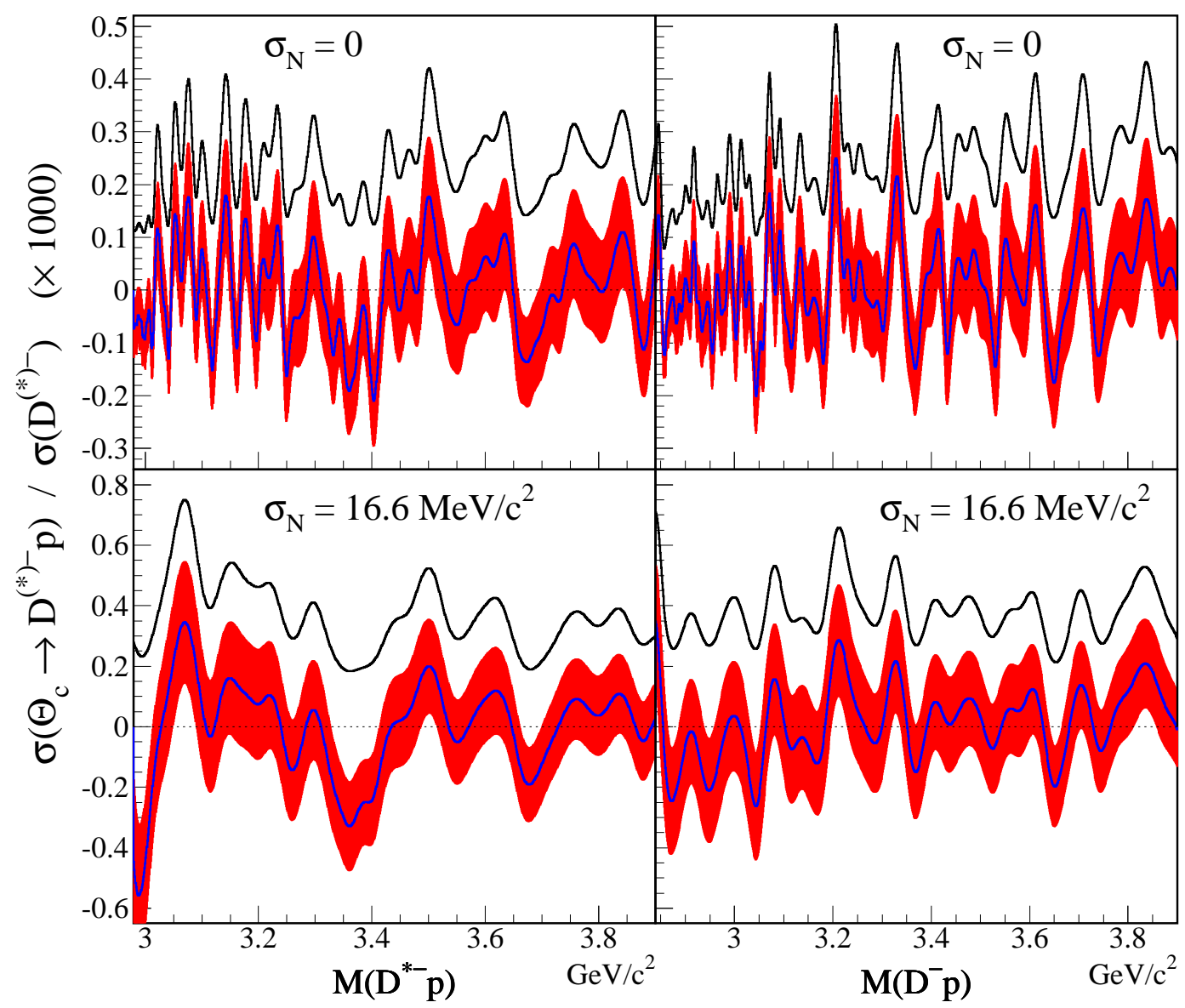

Fig. 7. Cross section times branching ratio results relative to single $D$ production. Left (right) plots show results for $\Theta_{c} \rightarrow D^{*-} p\left(\Theta_{c} \rightarrow D^{-} p\right)$. Top (bottom) plots show results for a natural width of $0\left(16.6 \mathrm{MeV} / c^{2}\right)$. The shaded region encompasses the $1 \sigma$ errors with the central value in the middle. The top curve shows the upper limit. Both include systematic uncertainties.

events to have $1<Q^{2}<100 \mathrm{GeV}^{2} / \mathrm{c}^{2}$ and $0.05<y<0.70$. From these samples, the production rate of $\Theta_{c}^{0}(3100)$ to $D^{*+}$ was found to be $1.58 \%(1.19 \%)$ for $\mathrm{H} 1$ (FOCUS). Scaling by the observed production rate of $1 \%$ at $\mathrm{H} 1$, the production rate of $\Theta_{c}^{0}(3100)$ relative to $D^{*+}$ should be $(1.19 / 1.58) \cdot 0.01=0.75 \%$ for FOCUS. For this FOCUS analysis, the $\Theta_{c}^{0}(3100)$ efficiency relative to $D^{*+}$ is $54 \%$. Therefore, from the FOCUS sample of $107,525 D^{*+}$ events, one would expect $107525 \cdot 0.0075 \cdot 0.54=435.5 \Theta_{c}^{0}(3100)$ events. This signal has been superimposed on the FOCUS data in Fig 8. This signal would be easily observable. The non observation by FOCUS is also consistent with published results from ALEPH [28] and ZEUS [29]. 
Table 1

Summary of upper limits (UL) on pentaquark yields and cross sections including systematic uncertainties. Results represent the maximum UL over the mass range shown in Figs. 3 and 7.

\begin{tabular}{cccc} 
Decay Mode & $\begin{array}{c}\text { Natural Width } \\
\left(\mathrm{MeV} / c^{2}\right)\end{array}$ & $\begin{array}{c}95 \% \text { CL UL } \\
\text { on Yield }\end{array}$ & $\begin{array}{c}95 \% \text { CL UL on } \\
\sigma\left(\Theta_{c}^{0}\right) \cdot \mathrm{BR}\left(\Theta_{c}^{0} \rightarrow D p\right) / \sigma(D)\end{array}$ \\
\hline \multirow{2}{*}{$\Theta_{c} \rightarrow D^{*-} p$} & 0 & 25 & $4.2 \times 10^{-4}$ \\
& 16.6 & 44 & $7.5 \times 10^{-4}$ \\
$\Theta_{c} \rightarrow D^{-} p$ & 0 & 31 & $5.0 \times 10^{-4}$ \\
& 16.6 & 41 & $7.1 \times 10^{-4}$
\end{tabular}

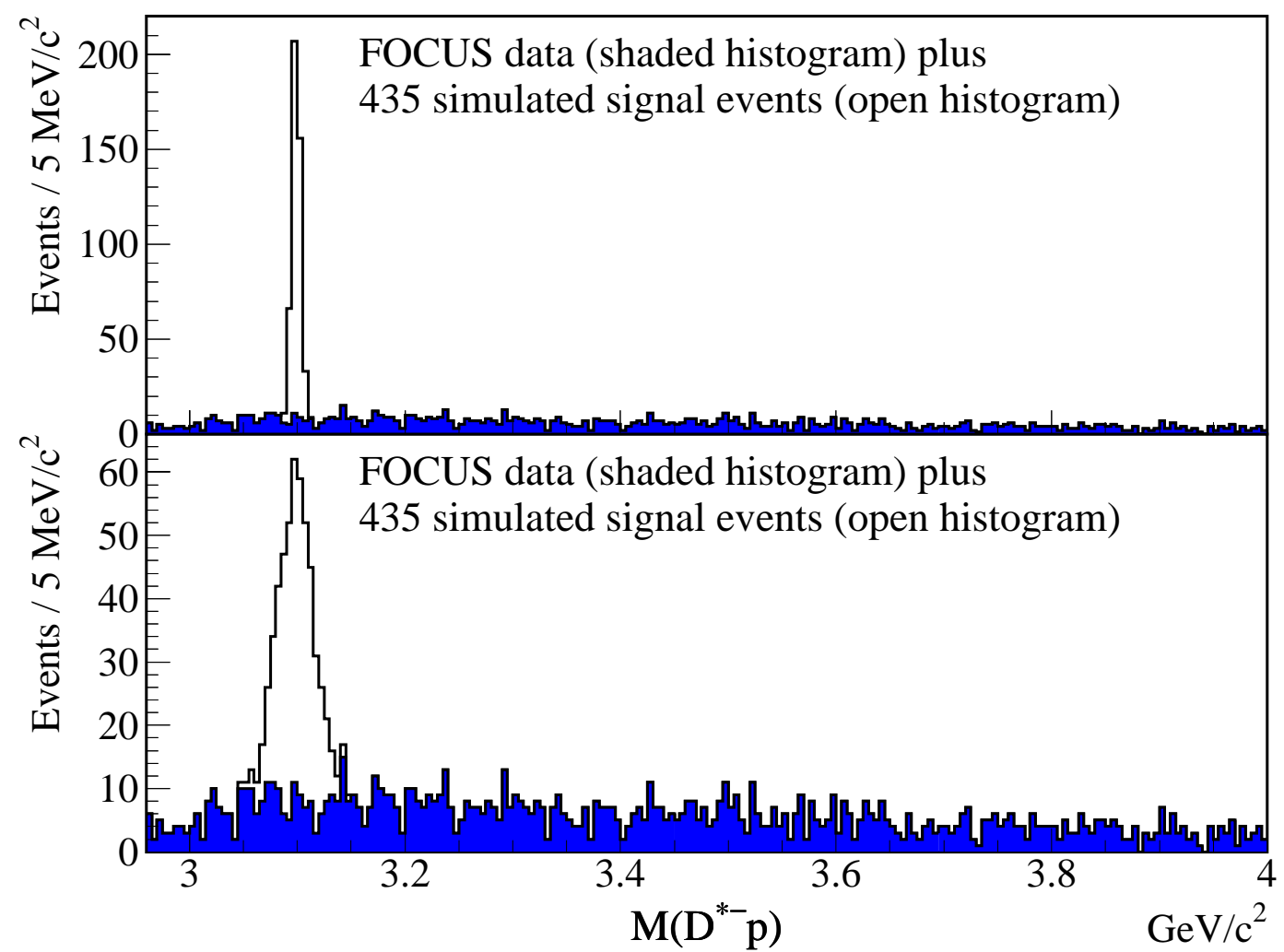

Fig. 8. The FOCUS data (from the left plot of Fig 2) plus a simulated Gaussian signal of 435 events based on the observed production at H1. The top plot shows the signal with a width due to experimental resolution $\left(\sigma=4.15 \mathrm{MeV} / c^{2}\right)$ only while the bottom plot shows the signal with a width due to the experimental resolution plus the maximum natural width (at 95\% CL) based on the H1 data $\left(\sigma=4.15 \oplus 16.6=17.1 \mathrm{MeV} / c^{2}\right)$.

\section{Acknowledgments}

We wish to acknowledge the assistance of the staffs of Fermi National Accelerator Laboratory, the INFN of Italy, and the physics departments of the 
collaborating institutions. This research was supported in part by the U. S. National Science Foundation, the U. S. Department of Energy, the Italian Istituto Nazionale di Fisica Nucleare and Ministero dell'Istruzione dell'Università e della Ricerca, the Brazilian Conselho Nacional de Desenvolvimento Científico e Tecnológico, CONACyT-México, the Korean Ministry of Education, and the Korean Science and Engineering Foundation.

\section{References}

[1] R. L. Jaffe, Phys. Rev. D 15 (1977) 267; R. L. Jaffe, Phys. Rev. D 15 (1977) 281; R. L. Jaffe, Phys. Rev. Lett. 38 (1977) 195; Erratum-ibid 38 (1977) 617.

[2] A. Chodos et al.., Phys. Rev. D 9 (1974) 3471; T. DeGrand et al.. Phys. Rev. D 12 (1975) 2060.

[3] H. J. Lipkin, Phys. Lett. B 195 (1987) 484.

[4] C. Gignoux, B. Silvestre-Brac, and J. M. Richard, Phys. Lett. B 193 (1987) 323.

[5] G. Karl and P. Zenczykowski, Phys. Rev. D 36 (1987) 3520.

[6] J. Leadri and B. Silvestr-Brac Phys. Rev. D 40 (1989) 2340.

[7] S. Fleck et al., Phys. Lett. B 220 (1989) 616.

[8] S. Zouzou and J. M. Richard, Few Body Syst. 16 (1994) 1.

[9] S. Takeuchi et al., Phys. Lett. B 318 (1993) 1.

[10] M. Genovese, et al., Phys. Lett. B 425 (1998) 171.

[11] F. Stancu, Phys. Rev. D 58 (1998) 111501.

[12] D. O. Riska and N. N. Scoccola, Phys. Lett. B 299 (1993) 338.

[13] Y. Oh, B. Y. Park, and D. P. Min, Phys. Rev. D 50 (1994) 3350; Y. Oh, B. Y. Park, and D. P. Min, Phys. Lett. B331 (1994) 362.

[14] C. K. Chow, Phys. Rev. D 51 (1995) 6327; C. K. Chow, Phys. Rev. D 53 (1996) 5108.

[15] E791 Collaboration, E. M. Aitala et al., Phys. Rev. Lett. 81 (1998) 44; E791 Collaboration, E. M. Aitala et al., Phys. Lett. B 448 (1999) 303.

[16] LEPS Collaboration, T. Nakano et al., Phys. Rev. Lett. 91 (2003) 012002; DIANA Collaboration, V. V. Barmin et al., Phys. Atom. Nucl. 66 (2003) 1715; CLAS Collaboration, S. Stepanyan et al., Phys. Rev. Lett. 91 (2003) 252001; SAPHIR Collaboration, J. Barth et al., Phys. Lett. B 572 (2003) 127; A. E. Asratyan et al., Phys. Atom. Nucl. 67 (2004) 682; CLAS Collaboration, 
V. Kubarovsky et al., Phys. Rev. Lett. 92 (2004) 032001; Erratum-ibid. 92 (2004) 049902; HERMES Collaboration, A. Airapetian et al., Phys. Lett. B 585 (2004) 213; SVD Collaboration, A. Aleev et al., hep-ex/0401024; COSYTOF Collaboration, M. Abdel-Bary et al., Phys. Lett. B 595 (2004) 127; ZEUS Collaboration, S. Chekanov et al., Phys. Lett. B 591 (2004) 7.

[17] M. Karliner and H. J. Lipkin, hep-ph/0307343, unpublished; R. Jaffe and F. Wilczek, Phys. Rev. Lett. 91 (2003) 232003; K. Cheung, Phys. Rev. D 69 (2004) 094029; P. Z. Huang et al., Phys. Rev. D 70 (2004) 034003; I. W. Stewart, M. E. Wessling, and M. B. Wise, Phys. Lett. B 590 (2004) 185.

[18] B. Wu and B. Q. Ma, Phys. Rev. D 70 (2004) 034025.

[19] S. Sasaki, Phys. Rev. Lett. 93 (2004) 152001.

[20] H1 Collaboration, A. Aktas et al., Phys. Lett. B 588 (2004) 17.

[21] H. Kim, S. H. Lee, and Y. Oh, Phys. Lett. B 595 (2004) 293; K. Maltmana, Phys. Lett. B 604 (2004) 175; D. Pirjol and C. Schat Phys. Rev. D 71 (2005) 036004 .

[22] FOCUS Collaboration, J. M. Link et al., Nucl. Instrum. and Meth. A 516 (2004) 364 .

[23] FOCUS Collaboration, J. M. Link et al., Nucl. Instrum. and Meth. A 484 (2002) 270.

[24] F. James and CN/ASD Group, Minuit - Function Minimization and Error Analysis - Reference Manual, Version 94.1, CERN (1994), unpublished.

[25] D. C. Carey et al., Decay TURTLE (Trace Unlimited Rays Through Lumped Elements): A Computer Program for Simulating Charged Particle Beam Transport Systems, Including Decay Calculations, SLAC-R-246, FERMILABPM-31, (1982), unpublished.

[26] T. Sjöstrand, Comp. Phys. Comm. 101 (1994) 232.

[27] FOCUS Collaboration, J. M. Link et al. , Phys. Lett. B 586 (2004) 11.

[28] ALEPH Collaboration, S. Schael et al., Phys. Lett. B 599 (2004) 1.

[29] ZEUS Collaboration, S. Chekanov et al., Eur. Phys. J. C 38 (2004) 29. 